\title{
Asexual Polyamory: Potential Challenges and Benefits
}

\author{
Dan Copulsky \\ Chicago, Illinois
}

\section{Introduction and Background}

Over the past two decades, a broad community has come together around the asexual identity (Hinderliter, 2009). While asexuality generally describes a sexual orientation in which a person does not experience sexual attraction toward anyone, specific experiences of asexual people vary considerably (Carrigan, 2011), and related identities are often considered part of the asexual spectrum or "ace" community (Chasin, 2015). Some members of the ace community also identify as polyamorous, participating in multiple romantic or intimate relationships that are not necessarily sexual (Scherrer, 2010).

One self-identified "relationship anarchist and celibate asexual" described the overlap between these two communities simply: "Given that polyamory is about wanting and being capable of having more than one romantic/emotionally significant relationship at the same time, it should be easy to understand that some asexuals are polyamorous" (Crosswell, 2013). Asexuals may also choose to be in polyamorous relationships in which they have a single partner, but that partner may have one or more additional relationships.

Some asexual people have observed that polyamory seems to be more popular as a relationship style among asexuals than it is in the mainstream. Another polyamorous asexual described the contemporary state of affairs this way: "No longer a whisper, polyamory has become the main relationship model for romantic asexuals. Every panel and discussion on relationship models includes a token part on monogamy because it's assumed that everyone already knows about polyamory" (Cerebus, 2014).

This article draws heavily on the personal narratives of asexual individuals to explore this intersection and focuses on ways in which polyamory can be particularly challenging or beneficial for those in the asexual community. These first-hand accounts come from posts that have been previously published online and from personal correspondence. Thirty-four Englishspeaking individuals were reached through a combination of the author's own networks and snowball sampling (through both the Facebook and Tumblr popular social media sites). All correspondents identified as either part of the asexual community or as partners with someone in this community. The quotes included herein represent themes that emerged from the broader collection of responses.

There is a need for scholarship that focuses on the intersection of asexuality with polyamory. For example, Prause and Graham (2007) and Brotto et al. (2010) discuss asexuals' relationships broadly, but do not address specific types of relationships. However, the present exploration builds on the work of both Klesse (2014), who investigated the varied ways that polyamorous identities are experienced, and Scherrer (2010), who explored the ways that 
asexuals define their relationships. Scherrer demonstrated that some asexuals are open to entering into polyamorous relationships.

\section{Potential Challenges of Asexual Polyamory}

Members of the asexual community have noted that there are ways in which polyamory can be particularly challenging for them (Cerberus, 2014; Morgie, 2015). These do not apply to every relationship, of course, but there are specific challenges that some asexuals may encounter with nonmonogamy. Asexuals also may face many of the same difficulties as others practicing polyamory, in addition to difficulties from identifying as asexual. As one writer described it, "Ace/Aro-spectrum ${ }^{1}$ people are subject to a lot of misunderstandings and stigmas already. Being ace/aro-spectrum AND poly can mean dealing with a lot more confusion and judgment from people who just don't 'get it'" (Morgie, 2015).

One challenge is that outsiders may misunderstand why asexuals are nonmonogamous. A correspondent who asked to be identified as Claire elaborated on this issue: "I don't like the way aces are only really included in the discussions around polyamory in the tone of 'Poly can be great for aces, that way they don't have to provide sex for their partner!' That is true, but there are... aces like me who are inclined to multiple deep and meaningful relationships." It seems to be important to many asexuals that people with other sexual identities understand their particular identities and motivations.

In a similar vein, outsiders who know that an individual is nonmonogamous, but who are not aware that that person is asexual, may make false assumptions about the person's sexuality. Another anonymous correspondent wrote that "Polyamory, swinging, and open relationships are often associated with sex and orgies by people who don't know much about the poly community."

This can also happen specifically within polyamorous communities where there are expectations about sexual interest and availability. Rhian Ruari Kerr described that "it can be challenging, however, in the poly community, when people expect you to be more open to sexual exploration than you want to be or are in any way set up or aligned to be.... There's an assumption in the poly community, at least in my experience, that 'poly' means 'available' in some manner."

A correspondent who asked to be identified as Smiles described another challenge that occurs "...when a sexual person and an asexual person have a closed romantic relationship but an open sexual relationship because the lack of sex between the two caused problems. I see this type of open relationship suggested a lot, even in cases where one of the people in the relationship would prefer to be in a monogamous relationship." Particularly as nonmonogamy becomes a more popular relationship style in the asexual community, it may be commonly prescribed even though such an arrangement may not work for all relationships.

Beyond this, asexuals may also be pressured or coerced by a partner into a nonmonogamous relationship. As Morgie described it, "Ace/aro-spectrum people run a risk of being coerced into poly when they really don't want it.... An ace or aro person should never feel 
like they are obligated to cave to others' desires" (2015). Asexuals may feel as though they must agree to a relationship style that they do not actually want.

Being nonmonogamous can also exacerbate relationship issues that asexuals already face. Morgie noted that "the problems in a relationship get magnified the more people are involved. So if there are unresolved issues, adding more people can just make things more complicated to resolve" (2015). An anonymous correspondent mentioned feelings of insecurity and jealousy in particular: "Aces who don't want to have sex but who have partners who have sexual relations with their other partner may feel left out or inadequate. It can be a source of jealousy." These could be difficult emotions that an asexual person already may struggle with.

Another anonymous correspondent commented on how it can be more difficult to navigate identities around multiple partnerships: "A last challenge is that being involved with multiple people means negotiating different levels of intimacy (sexual and otherwise) with each of them. This would be true whatever our sexual orientations, but I think for many asexual people these negotiations can be more tricky."

\section{Potential Benefits of Polyamory for Asexuals}

Members of the asexual community have also noted a number of ways in which polyamory can be particularly beneficial to them (Cerberus, 2014; Morgie, 2015; Scherrer, 2010). Again, these do not apply to every relationship. Asexuals may practice polyamory for many of the same reasons that others are polyamorous. For example, one may simply feel an innate desire to be in more than one intimate, loving relationship.

One of the most commonly cited reasons for asexuals to consider nonmonogamy is that it can allow their partners to get sexual desires met elsewhere when such desires are not met in the relationship or when meeting them is difficult. One correspondent, who asked to be identified as Laryssa, gave this description: "One of the main benefits of polyamory, specifically when it comes to asexual people, is when an asexual is dating an allosexual ${ }^{2}$, polyamory allows the allosexual to get the sex they generally desire without it becoming a strain on the allo/ace relationship."

While this dynamic benefits an asexual's partner more directly, it could also offer an asexual substantial relief from pressure to have sex. Charlie Mitchell described such a situation: "I think for a lot of ace people, it can take the pressure off when dating an allosexual person. If you are both open to polyamory and able to manage it, then it can be good that your partner has someone with whom they can have a sexual relationship."

This pressure to have sex could come from a partner, but an anonymous correspondent also described the way it could come from other places: "Even with a partner who is willing to forgo sex, in traditional monogamous relationships there is an overwhelming pressure from society/the mainstream media.... If you're not having sex, people either assume that you are, or become concerned that you are not." Thus, nonmonogamy can also alleviate social pressure or an internalized sense of how a relationship "is supposed to be." 
Another anonymous correspondent noted a very different way in which nonmonogamy was beneficial to them: "Polyamory is helpful in that it lets me explore the nuances of my desire for several different people. For a lot of my life I've had question marks surrounding my a/bi/sexuality, and while I still do, it's fascinating to be involved with multiple people of different genders, kink roles, and personalities." Rather than viewing nonmonogamy as a way to relieve particular burdens, this insight suggests that it can be active way to empower asexuals to explore desire or sexuality on their own terms.

Many also noted that the values emphasized in polyamorous communities could be particularly useful for asexuals. One writer described it this way: "The emphasis on good, consistent communication makes it easier for people of all orientations to express both their desires and their boundaries, and have these things respected" (Morgie, 2015). A number of correspondents specifically mentioned standards of communication, honesty, and openness.

Asexuals may also benefit especially from the flexible boundaries between intimate friendships and romantic relationships that nonmonogamy can make available. Smiles gave this description: "For some of us there is a blur between friendship and romance, and polyamory can help make that uncertainty less stressful. When I was in a monogamous relationship I was never sure whether or not my relationships with close friends were too intimate and crossed a line."

As Scherrer (2010, p.159) wrote, "Similar to those in polyamorous communities, asexual individuals are actively restructuring and rewriting their relationships, opening up possibilities for reimagining all of our lives." Many asexuals are finding benefits available to them in polyamory and other kinds of nonmonogamy. These benefits, along with the challenges previously discussed, highlight the importance of examining what polyamorous relationships look like specifically for the asexual community.

\section{Conclusion}

As is true for a wide range of diverse sexualities, polyamory is a relationship style that may be well suited for some asexual people but less so for others. Understanding the motivations that draw many asexuals to polyamory and the specific ways in which this kind of relationship can be difficult for them is a cornerstone both for providing culturally competent support and for conducting inclusive research. Three recommendations are offered below to help professionals and community members to better serve asexual and polyamorous individuals.

First, a clinician working with an asexual client should not assume what kinds of relationships the client may be a part of, or what the client's reasons are for choosing such relationships. The clinician should also remain sensitive to aspects of relationships that may be particularly emotional for specific clients.

Researchers studying nonmonogamy can be diligent in deciding what varieties of intimate relationships to be included in their work and communicating these decisions clearly throughout the research process. If a study is limited to looking at sexual relationships, potential participants should be aware of this limitation, and research reports should be clear that the 
particular study does not necessarily encompass the potential richness and diversity of polyamory.

Finally, professionals advocating for asexuals through writing or speaking should strive to better reflect the full scope of experiences and insights that are represented within this community. Researchers can continue examining these rich and diverse experiences with additional study. While this paper offers insights regarding potential challenges and benefits of asexual people in polyamorous relationships, much more research is needed that focuses on the overlap between these communities. Particularly, research utilizing larger sample sizes that explores challenges and benefits, as suggested here, is warranted and useful.

\section{Notes:}

1. Aromantism describes a romantic orientation in which a person does not experience romantic attraction. "Aro" is a common abbreviation for aromantic, and such identities are often considered "the aro spectrum."

2. In the asexual community, the word allosexual is used to describe people who do experience sexual attraction - that is, people who are not asexual. Allosexual is often abbreviated as "allo."

\section{References}

Brotto, L. Knudson, G., Inskip, J., Rhodes, K., and Erskine, Y. (2010). Asexuality: A mixed methods approach. Archives of Sexual Behavior, 39, 599-618.

Carrigan, M. (2011). There's more to life than sex? Difference and commonality within the asexual community. Sexualities, 14(4), 462478.

Cerberus. (2014). My Ace Poly Manifesto. Retrieved from

transpolyasexual.wordpress.com/2014/01/30 /my-ace-poly-manifesto/

Chasin, C. J. (2015). Making sense in and of the asexual community: Navigating relationships and identities in a context of resistance. Journal of Community \& Applied Social Psychology, 25(2), 167-180.
Crosswell, M. (2013, November 3). The Big, Fat Polyamorous Asexual Post. Retrieved from thethinkingasexual.wordpress.com/2013/11/ 02/the-big-fat-polyamorous-asexual-post/

Hinderliter, A. C. (2009). Asexuality: The history of a definition. Retrieved from asexualexplorations.net/home/history_of_de finition.html

Klesse. C. (2014). Polyamory: Intimate practice, identity, or sexual orientation. Sexualities, 17, 81-99.

Morgie. (2015). Her Name Is Gabe. Retrieved from hernameisgabe.tumblr.com

Prause, N. \& Graham, C. (2007). Asexuality: Classification and characterization. Archives of Sexual Behavior, 36, 341-356.

Scherrer, K. S. (2010). Asexual relationships: What does asexuality have to do with polyamory? Understanding non-monogamies, 154-159. London: Routledge. 